\title{
Isolation, synthesis and bioactivity studies of phomactin terpenoids
}

\author{
Yusuke Kuroda $\mathbb{1}^{1}$, Karen J. Nicacio $\mathbb{1}^{2}$, Ildefonso Alves da Silva-Jr $\mathbb{1}^{3}$, Paul R. Leger', Stanley Chang', \\ Juliana R. Gubiani', Victor M. Deflon², Nozomu Nagashima1, Alexander Rode', Katherine Blackford', \\ Antonio G. Ferreira ${ }^{4}$, Lara D. Sette ${ }^{5}{ }^{5}$, David E. Williams ${ }^{6}$, Raymond J. Andersen ${ }^{6}$, Sonia Jancar ${ }^{3 \star}{ }^{3 \star}$ \\ Roberto G. S. Berlinck ${ }^{2 \star}$ and Richmond Sarpong ${ }^{10}{ }^{1 \star}$
}

\begin{abstract}
Studies of secondary metabolites (natural products) that cover their isolation, chemical synthesis and bioactivity investigation present myriad opportunities for discovery. For example, the isolation of novel secondary metabolites can inspire advances in chemical synthesis strategies to achieve their practical preparation for biological evaluation. In the process, chemical synthesis can also provide unambiguous structural characterization of the natural products. Although the isolation, chemical synthesis and bioactivity studies of natural products are mutually beneficial, they are often conducted independently. Here, we demonstrate the benefits of a collaborative study of the phomactins, diterpenoid fungal metabolites that serve as antagonists of the platelet activating factor receptor. Our isolation of novel phomactins has spurred the development of a bioinspired, unified approach that achieves the total syntheses of six congeners. We also demonstrate in vitro the beneficial effects of several phomactins in suppressing the rate of repopulation of tumour cells following gamma radiation therapy.
\end{abstract}

T he study of secondary metabolites (natural products) and their biological function often begins with their isolation and characterization from a producing organism, followed by screening of the isolates for bioactivity. Secondary metabolites that reveal interesting properties may then be chemically prepared to obtain larger quantities of materials to facilitate additional bioactivity studies. Syntheses of these often complex molecules may also be used to support or revise their structural characterization in cases where the structure cannot be unambiguously ascertained ${ }^{1}$. Finally, chemical synthesis may also provide derivatives of the natural isolate not accessible through biosynthesis or late-stage modification that might possess unmatched bioactivity ${ }^{2,3}$. A medicinal chemistry campaign may then be undertaken to obtain a promising drug candidate ${ }^{4}$. Given this holistic approach to identifying novel bioactive entities and pharmaceuticals based on natural products, it is surprising that the isolation, synthesis and functional assays of secondary metabolites are seldom undertaken contemporaneously. Such an approach will undoubtedly create a 'feedback loop' that would accelerate the discovery process and broaden its scope at each stage. Herein, we report a collaborative approach to studying natural products in the phomactin family that spans isolation, chemical synthesis and investigation of bioactivity. Our studies have (1) yielded novel members of these marine diterpenoids that are produced by a unique fungal strain, (2) established a chemical synthesis strategy that achieves the shortest total syntheses of several congeners from carvone, and (3) led to the discovery of the beneficial function of several phomactins in suppressing the rate of repopulation of tumour cells after gamma radiation therapy.

The phomactins (see Fig. 1a for representative members) are diterpenoids first isolated in $1991^{5}$ from cultures of the fungus
Phoma sp. Additional phomactins, including phomactins $B-G^{6,7}$, phomacta-1(14),3,7-triene and Sch $49027^{\circ}$, are produced by the same species and were reported between 1993 and 1995. Subsequent to these reports, Koyama's analysis of a culture from an unidentified fungus (MPUC 046) yielded the structurally related compounds that were named phomactins $\mathrm{H}^{9}, \mathrm{I}-\mathrm{J}^{10}, \mathrm{~K}-\mathrm{M}^{11}$ and $\mathrm{N}-\mathrm{P}^{12}$. Our collection and analysis of culture medium of the marine-derived fungus Biatriospora sp. (CBMAI 1333) ${ }^{13}$ has now yielded phomactins Q-V (1-6, Fig. 1a), resulting in the isolation, so far, of 27 phomactins. The structures of the newly isolated phomactins 1-6 were elucidated by spectroscopic analysis using one- (1D) and two-dimensional (2D) NMR techniques and mass spectrometry. The structure of phomactin S (3) was unambiguously determined by X-ray diffraction analysis (Supplementary Fig. 39), which also corroborated the absolute configuration we have assigned to this compound. Given its unusual framework and that the small amount of isolated material decomposed over time, the structure of phomactin T (4) was more challenging to establish. It was ultimately secured using 2D NMR techniques (Supplementary Table 17) as well as by a comparison of its spectral data with that of material prepared through chemical synthesis (see the 'Chemical synthesis' section for details).

The phomactins, particularly phomactin A (7, Fig. 1b), emerged from an assay-guided fractionation of fungal metabolites to identify platelet activating factor receptor (PAFR) ${ }^{14}$ antagonists. Notably, 7 had no effect on collagen-induced platelet aggregation. Blocking PAFR has proven effective in experimental models of asthma, renal disease, sepsis and pancreatitis, among other diseases ${ }^{15}$. It has been shown previously that combination therapy employing PAFR antagonists and radiation or chemotherapy is particularly effective in inhibiting human melanoma (B16F10 and SKmel137 cells,

'Department of Chemistry, University of California, Berkeley, CA, USA. ${ }^{2}$ Instituto de Química de São Carlos, Universidade de São Paulo, São Carlos, SP, Brazil. ${ }^{3}$ Departamento de Imunologia, Instituto de Ciências Biomédicas, Universidade de São Paulo, São Paulo, SP, Brazil. ${ }^{4}$ Departamento de Química, Universidade Federal de São Carlos, São Carlos, SP, Brazil. ${ }^{5}$ Departamento de Bioquímica e Microbiologia, Instituto de Biociências, Universidade Estadual Paulista Júlio de Mesquita Filho, Campus Rio Claro, Rio Claro, SP, Brazil. ' ${ }^{6}$ epartments of Chemistry and Earth, Ocean \& Atmospheric Sciences, University of British Columbia, Vancouver, British Columbia, Canada. *e-mail: sojancar@icb.usp.br; rgsberlinck@iqsc.usp.br; rsarpong@berkeley.edu 


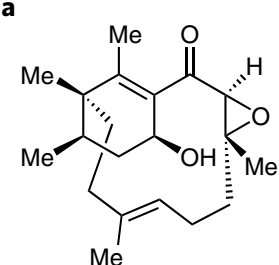

Phomactin Q (1)<smiles>C=C1C(C(=O)C2OC2C)=CCC(C)(C/C(C)=C/CC[C@H]2OC2C)[C@@H]1C</smiles>

Phomactin R (2)

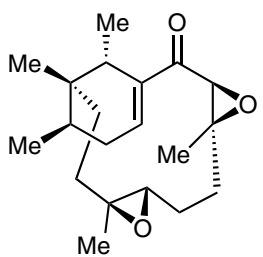

Phomactin S (3)

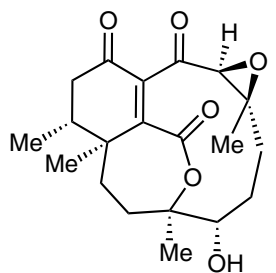

Phomactin T (4)

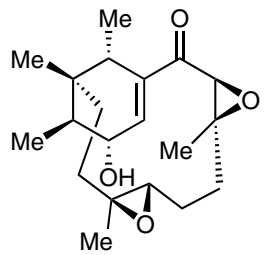

Phomactin U (5)

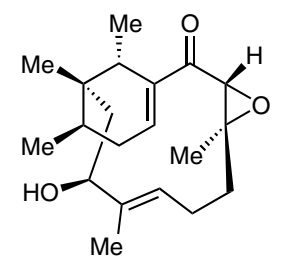

Phomactin V (6)

b

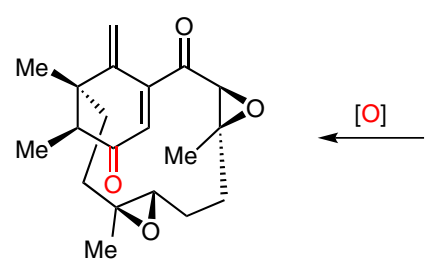

Phomactin K (13)<smiles>C=C1C(C(=O)C2O[C@H]2CCC2OC2(C)CC2CCCCC2C)=CCC1(C)C</smiles>

Phomactin P (12)

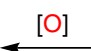<smiles>C=C1C(C(=O)C2O[C@H]2C)=CCC(C)C1(C)CC(C)=CCC[C@H]1CO1</smiles>

Phomactin R (2)<smiles>CCCCC=C(C)CCC=C(C)CCC=C(C)CCCC</smiles>

Geranylgeranyl pyrophosphate

(8)<smiles>C[C@@H]1O[C@H]1C(=O)C1=C2C(=O)O[C@@](C)(CC[C@@H](C)CC1=O)CC[C@@H](O)C2(C)C</smiles>

Phomactin T (4)

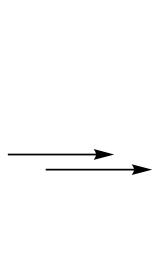

Phomacta-1(14),3,7-triene

(9)

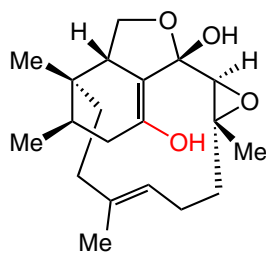

Sch 49027 (14) [proposed structure]

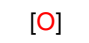

$+$

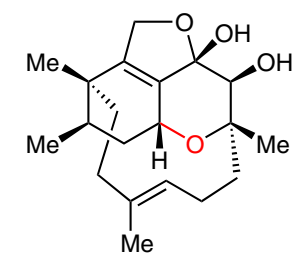

Phomactin A (7)
Phomactin E (11)
<smiles>C=CCC(=O)OC</smiles>

(S)-(+)-carvone carvone core

(19)<smiles>C=C1C(C=O)=CCC(C)[C@]1(C)COc1ccccc1</smiles>

17

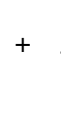<smiles>C1CCCCC1</smiles><smiles>C=C(C)COC</smiles><smiles>CC(C)=CC=C(C)C</smiles>

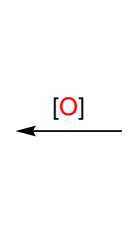

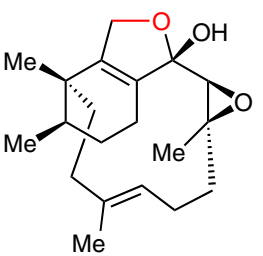

Phomactin G (15)<smiles>C=C1C(C(O)/C=C(\C)CC/C=C(/C)CC2CCCCC2C)=CCC1C</smiles>

Common synthetic intermediate (16)

Fig. 1 | Members of the phomactin family and retrosynthetic analysis based on the plausible biosynthetic pathway. a, Newly isolated phomactin congeners. b, Plausible biosynthetic pathways for the phomactins. c, Retrosynthesis of common synthetic intermediate 16.

respectively) and TC-1 (tissue culture number 1) murine carcinoma regrowth ${ }^{16}$. PAFR antagonists could therefore serve as adjuvants in cancer therapy by inhibiting the interaction between PAFR and PAF-like molecules generated during chemotherapy or irradiation ${ }^{17}$.

In addition to their function as PAFR antagonists, the intriguing structures of the phomactins, which feature a bicyclo[9.3.1]pentadecane core comprised of a cyclohexenyl ring and macrocyclic 'strap' that bridges the ring, have inspired interest in these molecules from synthetic chemists for over 20 years. The many inventive approaches to the synthesis of the phomactins have been reviewed recently ${ }^{18}$. So far, six total syntheses of phomactin congeners have been reported. Four of these syntheses (of phomactins A, G and B2) were achieved in racemic form, ranging in step count from 18 to 37 (longest linear sequence, LLS) starting from commercially available materials ${ }^{19-22}$. Only two reports of total syntheses culminating in enantioenriched phomactin natural products have appeared: in 1996, Yamada and co-workers reported an enantioselective synthesis of phomactin D in 36 steps (LLS) and, in 2003, Halcomb and colleagues disclosed an enantiospecific synthesis of phomactin A in 27 steps (LLS) from $(R)-(+)$-pulegone ${ }^{23,24}$.

An unmet challenge in the chemical synthesis of the phomactins is to devise a strategy that leads to all the structural types in the family-ideally from a common late-stage intermediate. To address this challenge, our retrosynthetic analysis, informed by the existing 
<smiles>C=C(C)[C@H]1CC=C(C)C(=O)C1</smiles>

(S)-(+)-carvone (19)
1) $m$-CPBA (98\% yield, 1:1 d.r.)

2) $\mathrm{Cp}_{2} \mathrm{TiCl}_{2}, \mathrm{Zn}$

(66\% yield, 2:3 d.r.)

3) $\mathrm{PhSSPh}, \mathrm{Bu}_{3} \mathrm{P}$ ( $91 \%$ yield)<smiles>CC1=CCC2C[C@]1(Cc1ccccc1)[C@@]2(C)O</smiles>

20

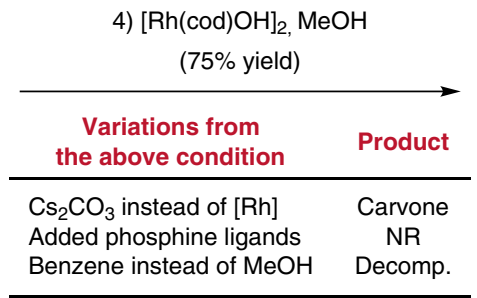<smiles>CC1=CCC(C)[C@](C)(Cc2ccccc2)C1=O</smiles>

21

5) $\left(\mathrm{NH}_{4}\right)_{6} \mathrm{Mo}_{7} \mathrm{O}_{24}$ $\mathrm{H}_{2} \mathrm{O}_{2}$ (95\% yield)

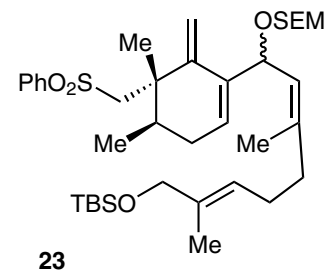

9) $18, t$-BuLi (89\% yield, 1:1 d.r.) 10) SEMCI (88\% yield)<smiles>C=C1C(C=O)=CCC(C)[C@@]1(C)CSc1ccccc1</smiles>

17

\author{
6) MeLi \\ 7) Burgess reagent \\ (86\% yield, 2 steps) \\ 8) $\mathrm{SeO}_{2}$ (56\% yield)
}<smiles>CC1=CCC(C)[C@](C)(CSc2ccccc2)C1=O</smiles>

22 23 11) TBAF 12) $\mathrm{MsCl}, \mathrm{LiBr}$<smiles>[M]O[C@@H](/C=C(\C)CC/C=C(\C)CBr)C1=CC[C@@H](C)[C@@](C)(CS(=O)(=O)Oc2ccccc2)C1=C</smiles>

24

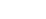

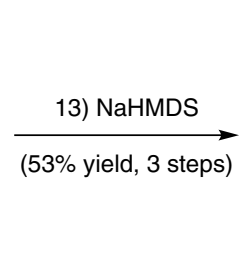

(1)

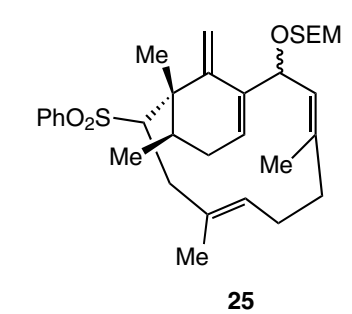

14) $\mathrm{Na} \bullet \mathrm{Hg}$

(75\% yield, $86 \%$ brsm)
15) TBAF, DMPU
16) $\mathrm{MnO}_{2} ; \mathrm{NaBH}_{4}, \mathrm{CeCl}_{3}$ (56\% yield, 2 steps, $13: 1$ d.r.)

25

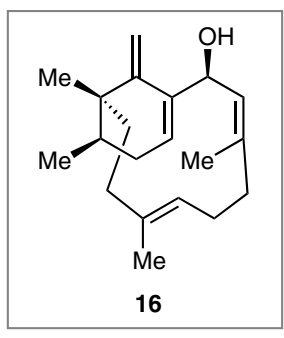

Fig. 2 | Synthesis of common intermediate 16. m-CPBA, meta-chloroperoxybenzoic acid; $\mathrm{Cp}_{2} \mathrm{TiCl}_{2}$, bis(cyclopentadienyl)titanium dichloride; cod, 1,5-cyclooctadiene; SEM, 2-(trimethylsilyl)ethoxymethyl; TBAF, tetra-n-butylammonium fluoride; MsCl, methanesulfonyl chloride; NaHMDS, sodium bis(trimethylsilyl)amide; DMPU, 1,3-dimethyltetrahydropyrimidin-2(1H)-one; BINAP, 2,2'-bis(diphenylphosphino)-1,1'-binaphthyl; NR, no reaction; brsm, based on recovered starting material.

syntheses, took inspiration from the proposed biosynthesis of these intriguing fungal secondary metabolites (Fig. 1b). Like other diterpenoids, the phomactin natural products are believed to arise from geranylgeranyl pyrophosphate (8), which is advanced to phomacta1(14),3,7-triene (9) through a series of carbocation-initiated cyclization events and attendant methyl and hydride shifts. The feasibility of these biosynthetic transformations is supported by ${ }^{13} \mathrm{C}$ labelling and density functional theory calculations ${ }^{25,26}$. Although the biosynthesis of the metabolites further down the biosynthetic pathway from 9 (Fig. 1b) remains largely speculative, the oxygenation pattern of these downstream congeners suggests that $\mathbf{1 0}$ may serve as a branching point in their biosynthesis. For example, a stereoselective hydroxylation (type A oxidation, Fig. 1b) at C15 in $\mathbf{1 0}$ would lead to phomactin E (11) and subsequently phomactin R (2), from which phomactin $\mathrm{P}(12)$ and phomactin $\mathrm{K}$ (13) may arise through a series of additional enzymatic oxidations. On the other hand, phomactin A (7), Sch 49027 (14) and phomactin T (4) probably arise from phomactin $G(15)$ via an alternative pathway starting with the selective oxidation of 10 (type B oxidation). To emulate P450-mediated chemoselective oxygenations in the biosynthesis of phomactins, we designed a suitably oxygenated phomactatriene derivative (16) as a common chemical synthesis intermediate (Fig. 1c). The choice of $\mathbf{1 6}$ hinged on maintaining the same overall oxidation level as $\mathbf{1 0}$ so as to facilitate access to the majority of the phomactin congeners. By inverting the oxidation state at $\mathrm{C} 2$ (see 9, Fig. 1b, for numbering) and $\mathrm{C} 15$ of $\mathbf{1 6}$ relative to $\mathbf{1 0}$ with the installation of a hydroxyl group at $\mathrm{C} 2$, we were poised to site-selectively direct the downstream oxygenation events that would have otherwise been difficult. In our envisioned bioinspired sequence, a series of C2 hydroxy-directed epoxidations and subsequent epoxide openings from 16 could yield divergent syntheses of many of the phomactin natural products. Oxidized phomactatriene derivative $\mathbf{1 6}$ would arise from cyclohexenyl fragment 17 and vinyl iodide 18, building on the pioneering studies of Yamada to construct the macrocyclic strap ${ }^{23}$.

Our analysis of the previous syntheses of the phomactins indicates to us that while inventive and efficient syntheses of the macrocyclic strap have been achieved, a significant investment of chemical steps has gone into preparing the densely functionalized cyclohexenyl-derived portion of the phomactins (especially for syntheses in enantioenriched form). Therefore, gains in efficiency for any new synthesis of the phomactins will primarily arise by identifying a shorter and more efficient synthetic sequence to the cyclohexenylderived fragment adorned with the strategically disposed functional groups necessary for the preparation of congeners. In a key recognition for our synthesis, we saw methylidene cyclohexene 17 (bearing challenging vicinal tertiary and quaternary carbon centres) arising from $(S)-(+)$-carvone (19), an abundant terpene feedstock available as either antipode (Fig. 1c). Although carvone has been extensively employed as a starting material for synthesis, strategies that exploit the rearrangement of its core framework to access other cyclohexenyl derivatives, of which our recent work is representative ${ }^{27}$, are rare. We sought to employ such a rearrangement of the core framework of $(S)$ - $(+)$-carvone that subsumes the isopropenyl group to provide rapid access to 17 . 


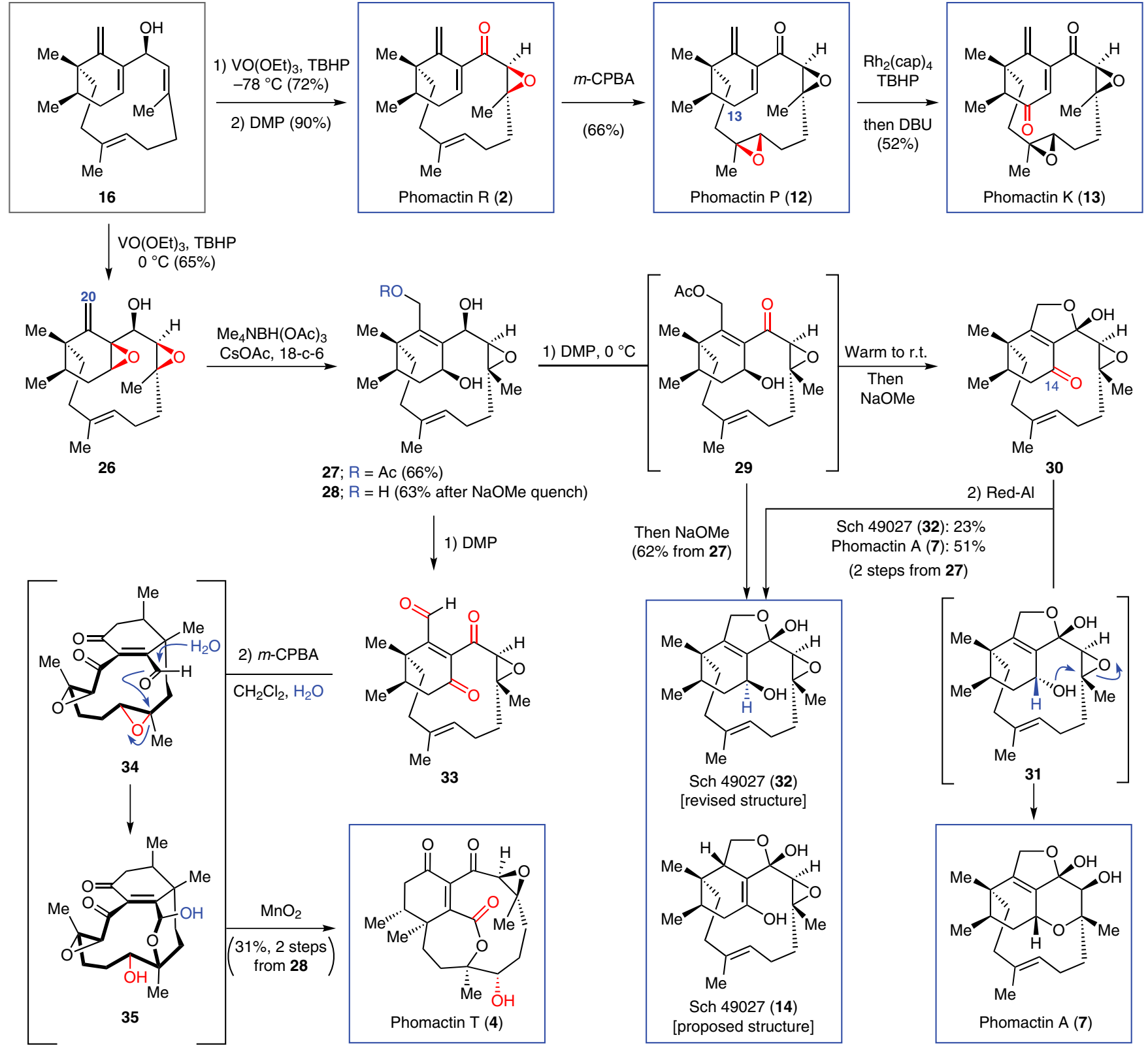

Fig. 3 Completion of the total syntheses of phomactins A, K, P, R and T and Sch 49027. TBHP, tert-butyl hydroperoxide; DMP, Dess-Martin periodinane; cap, caprolactamate; DBU, 1,8-diazabicyclo(5.4.0) undec-7-ene; Red-Al, sodium bis(2-methoxyethoxy)aluminum hydride; 18-c-6, 18-crown-6; r.t., room temperature.

\section{Results and discussion}

Chemical synthesis. Our synthetic studies commenced with the preparation of methylidene cyclohexene 17 (Fig. 2). Following the precedent of ref. ${ }^{28}$, the cyclobutanol moiety was installed through epoxidation of $(S)-(+)$-carvone, followed by $\mathrm{Ti}(\mathrm{III})$-mediated reductive coupling of the epoxide and carbonyl functional groups to provide a 2:3 ratio of cyclobutanol diastereomers, of which the minor was carried forward. Mitsunobu-type displacement of the resulting primary hydroxyl group gave phenyl sulfide 20. With cyclobutanol 20 in hand, we investigated the opening of the cyclobutanol to install the angular methyl group (C19) of the phomactins. Using an adaptation of our recently reported protocol ${ }^{27}$ that builds on precedent from refs 29,30 , exposure of $\mathbf{2 0}$ to catalytic [ $\mathrm{Rh}(\operatorname{cod})$ $\mathrm{OH}]_{2}$ in $\mathrm{MeOH}$ effects the desired $\mathrm{C}-\mathrm{C}$ bond cleavage to afford cyclohexene 21 in $75 \%$ yield. It is worth noting that (1) exposure of 20 to inorganic bases such as $\mathrm{Cs}_{2} \mathrm{CO}_{3}$ only promoted Grob fragmentation to give carvone, (2) addition of phosphine ligands including $\mathrm{Ph}_{3} \mathrm{P}, n-\mathrm{Bu}_{3} \mathrm{P}$ and BINAP completely inhibited reactivity, and (3) the use of $\mathrm{MeOH}$ as solvent was singularly effective. In this way, an overall rearrangement of the carvone core framework to integrate the isopropenyl group was achieved via $\mathbf{2 0}$ to furnish $\mathbf{2 1}$ . Oxidation of the phenyl sulfide moiety of $\mathbf{2 1}$ gave phenyl sulfone 22, which was treated with MeLi followed by the Burgess dehydrating reagent to append $\mathrm{C} 20$ of the phomactins. Allylic oxidation of the vinylic methyl group was accomplished using $\mathrm{SeO}_{2}$ to afford aldehyde 17. 1,2-Addition of a vinyl lithium nucleophile derived from known vinyl iodide $\mathbf{1 8}^{23}$ gave a bis-allylic alcohol (1:1 mixture of diastereomers) that was protected with a 2-(trimethylsilyl)ethoxymethyl (SEM) group to afford 23 . Treatment of 23 with TBAF unveiled the primary hydroxyl, which was converted to allylic bromide 24 via the mesylate. Macrocyclization of sulfone/allylic bromide $\mathbf{2 4}$ was effected by treatment with NaHMDS (in line with 
Table 1 | PAFR-inhibitory concentration $\left(\mathrm{IC}_{50}\right)$ of phomactins $(\mu \mathrm{M})$

\begin{tabular}{ll} 
Phomactins & IC $_{50}$ \\
\hline Phomactin A & 3.8 \\
Phomactin F & 2.7 \\
Phomactin I & 3.2 \\
Phomactin P & 3.0 \\
Phomactin R & 2.5 \\
Phomactin S & 2.8 \\
Phomactin U & 10.0 \\
Phomactin V & 3.1 \\
WEB 2170 & 3.2 \\
\hline
\end{tabular}

Concentration of phomactins or WEB 2170 (in $\mu$ M) that reduced $50 \%$ of the response to $10 \mathrm{nM}$ of $\mathrm{CPAF}\left(\mathrm{IC}_{50}\right)$. Inhibitory doses were generated using three-parameter non-linear regression analysis from $n=3$ independent experiments (see the 'Biological assays' and 'Statistical analysis' sections for more details). CPAF, carbamoyl-PAF (1-hexadecyl-2-N-methylcarbamoyl glycerophosphocholine)

\section{Table 2 | Inhibition of tumour cell repopulation by phomactin} congeners

\begin{tabular}{ll} 
Phomactins & \% inhibition of RLU* \\
\hline Phomactin A & $55 \pm 11$ \\
Phomactin R & $83 \pm 7$ \\
Phomactin S & $68 \pm 2$ \\
Phomactin P & $29 \pm 5$ \\
Phomactin U & $34 \pm 5$ \\
Phomactin F & $77 \pm 3$ \\
Phomactin V & $47 \pm 2$ \\
Phomactin I & $49 \pm 6$ \\
WEB 2170 & $71 \pm 2$ \\
\hline
\end{tabular}

*\% inhibition of RLU by $10 \mu \mathrm{M}$ of phomactin compounds or WEB 2170 relative to untreated control irradiated at $8 \mathrm{~Gy}$. Data are from $n \geq 3$ independent experiments and are presented as mean \pm standard error of the mean (s.e.m.).

the precedent of ref. ${ }^{23}$ ) to afford 25. Reductive removal of the sulfone group, cleavage of the SEM group with TBAF in $\mathrm{DMPU}^{31}$, and oxidation of the resulting secondary hydroxyl followed by diastereoselective $\mathrm{NaBH}_{4}$ reduction gave 16, which serves as a common synthetic intermediate to phomactin natural products in each of the known structural classes.

With key intermediate $\mathbf{1 6}$ in hand, we focused our attention on the syntheses of phomactins $\mathrm{R}, \mathrm{P}$ and $\mathrm{K}$. Hydroxyl-directed epoxidation of 16 with $\mathrm{VO}(\mathrm{OEt})_{3}$ (ref. ${ }^{32}$ ) and $\mathrm{TBHP}$ at $-78^{\circ} \mathrm{C}$ followed by oxidation of the hydroxyl group gave phomactin $\mathrm{R}$ (2, Fig. 3). The use of $\mathrm{VO}(\mathrm{OEt})_{3}$ was critical to achieving good diastereoselectivity in the epoxidation. In line with the presumed biosynthesis of the phomactins, epoxidation of phomactin R (2) using $m$-CPBA yields phomactin $\mathrm{P}$ (12). Furthermore, oxidation of the allylic $\mathrm{C} 13$ position of $\mathbf{1 2}$ using $\mathrm{Rh}_{2}(\mathrm{cap})_{4}$ (ref. ${ }^{33}$ ) and TBHP affords phomactin $\mathrm{K}$ (13).

Alternatively, a double epoxidation of 16 occurs following its exposure to $\mathrm{VO}(\mathrm{OEt})_{3}$ and excess $\mathrm{TBHP}$ at $0{ }^{\circ} \mathrm{C}$ to give bis-epoxide 26, setting the stage for a crucial epoxide opening to install the requisite hydroxy group at C20 of phomactins A, T and Sch 49027. After extensive experimentation, we found that treatment of $\mathbf{2 6}$ with $\mathrm{Me}_{4} \mathrm{NBH}(\mathrm{OAc})_{3}$ (ref. ${ }^{34}$ ) in the presence of CsOAc and 18-crown-6 initiated $\mathrm{S}_{\mathrm{N}} 2^{\prime}$ epoxide opening with acetate as the nucleophile to give diol $\mathbf{2 7}$ or triol $\mathbf{2 8}$ (when the acetyl group was cleaved using a $\mathrm{NaOMe}$ quench). With the oxygen installed at C20, we proceeded to synthesize phomactin A. Oxidation of diol 27 using the DessMartin periodinane reagent at room temperature, followed by cleavage of the acetyl group using $\mathrm{NaOMe}$, leads to ketone $\mathbf{3 0}$ where hemi-ketal formation occurs spontaneously. It is of note that exclusive formation of keto-alcohol $\mathbf{2 9}$ was observed when the reaction temperature was kept at $0^{\circ} \mathrm{C}$. The final step required stereoselective reduction of the $\mathrm{C} 14$ ketone in the presence of the labile hemi-ketal. An attempted hydroxyl-directed reduction using $\mathrm{Me}_{4} \mathrm{NBH}(\mathrm{OAc})_{3}$ (ref. ${ }^{35}$ ) gave complex mixtures, whereas, surprisingly, $\mathrm{NaBH}_{4}$ or $\mathrm{LiAlH}_{4}$ brought about the exclusive formation of a compound with analytical data fully consistent with that reported for Sch 49027. However, our analysis of the spectral data reported for Sch 49027 indicated that it was more consistent with the allylic alcohol structure obtained from the reduction step (that is, 32) and not the unusual enol 14. Because a pathway to convert 32 to 14 is not obvious, we propose that the structure of Sch 49027 should be revised to 32. Allylic alcohol 32 was also obtained in $62 \%$ yield when ketoalcohol 29 was treated with $\mathrm{NaOMe}$, further corroborating its structure. Notably, reduction of ketone $\mathbf{3 0}$ with Red-Al affords a 2.2:1 mixture of phomactin A (7) and 32. Phomactin A probably arose from diastereoselective carbonyl reduction of $\mathbf{3 0}$ to give $\mathbf{3 1}$ followed by cyclization of the resultant hydroxyl onto the epoxide. To access phomactin T, triol 28 was oxidized to keto-aldehyde 33 using the Dess-Martin periodinane reagent. On exposure of 33 to $m$-CPBA, lactol 35 formed directly through a cascade presumably initiated by stereoselective epoxidation of the double bond followed by incorporation of the hemi-acetal in 34. Although this transformation effectively constructs the phomactin $\mathrm{T}$ framework, the resulting lactol proved unstable and slowly decomposed. Therefore, a superior process was developed wherein the epoxidation step was telescoped with a $\mathrm{MnO}_{2}$ oxidation to afford phomactin $\mathrm{T}$ (4). In summary, total syntheses of phomactin A (20 steps), phomactin K (20 steps), phomactin P (19 steps), phomactin R (18 steps), phomactin T (20 steps) and Sch 49027 (19 steps) were achieved from $(S)-(+)$-carvone. In the process, the structure of phomactin $\mathrm{T}$ was confirmed and the structure of Sch 49027 was revised to 32. This synthetic work set the stage for our bioactivity studies.

Biological assays. Activation of PAFR in tumour cells through chemo- or radiotherapy activates intracellular programs that promote their survival and proliferation ${ }^{36}$. It is therefore expected that combining radiotherapy with PAFR antagonists could provide an effective combination therapy for reducing tumour cell proliferation. En route to this eventual goal, we evaluated the PAFR antagonist activities of several of the phomactins obtained from cultures of Biatriospora sp. (CBMAI 1333) or through chemical synthesis. Isolated and chemically synthesized phomactins were screened for PAFR antagonist activity in human epidermal carcinoma (KB) cells transfected with PAFR (KBP) by measuring interleukin 8 (IL-8) concentrations (see Methods and Supplementary Fig. 40a,b). A halfmaximum inhibitory concentration $\left(\mathrm{IC}_{50}\right.$ ) of $3.2 \mu \mathrm{M}$ was obtained for the PAFR antagonist WEB 2170 (Supplementary Fig. 40c), which served as a control. This $\mathrm{IC}_{50}$ value is in agreement with the previously determined potency for this compound in a human breast cancer cell line ${ }^{37}$. Table 1 shows the $\mathrm{IC}_{50}$ values of various phomactins as PAFR antagonists. Phomactins A, S, P, F, V and I were found to be as potent as WEB 2170, displaying $\mathrm{IC}_{50}$ values between 2.8 and $3.2 \mu \mathrm{M}$, within the range of previously determined values ${ }^{5,8}$. Notably, phomactin R emerged as the most potent of the phomactins, with an $\mathrm{IC}_{50}$ value of $2.5 \mu \mathrm{M}$. At the concentrations employed in this study, none of the phomactins was found to adversely affect cell viability as determined using the lactate dehydrogenase (LDH) cytotoxicity assay ${ }^{38}$. Overall, these in vitro studies reveal bioactivities for the phomactins that approach and in some cases surpass those of an 
established PAFR antagonist (WEB 2170). However, a more comprehensive comparison to other phomactins such as phomactin $\mathrm{D}$ using the same assay will need to be conducted. Also, given the limited data set, no definitive structure-activity relationships (SARs) for the phomactins could be gleaned from the results that we have so far obtained. A summary of some SAR trends that appear to be emerging are summarized in the Supplementary Information. In addition to reducing the viability of tumour cells, gamma irradiation also induces the over-production of PAFR ligands, which, by a direct effect or by enhancing prostaglandin E2 formation, promote tumour repopulation ${ }^{39}$. The investigation of the phomactins as inhibitors of tumour cell repopulation was planned only at the completion of our PAFR antagonist assays, and required additional amounts of the natural products that had to be procured by both re-isolation from the producing fungus and chemical synthesis. We used an in vitro assay to investigate the ability of several phomactin natural products to inhibit the repopulation of murine lung carcinoma TC-1 cells following radiation (see 'Biological assay' section for details). Consistent with the PAFR antagonist activity shown in Table 1, most compounds induced significant inhibition of TC-1 repopulation, tracking closely with their PAFR antagonist activity. Phomactin R showed the highest TC-1 repopulation inhibitory activity in comparison to the other phomactins (Table 2), consistent with its higher PAFR antagonist activity. Similarly, phomactin U showed the lowest repopulation inhibition activity, which correlates with its poor PAFR antagonist activity. Overall, our results suggest that irradiated TC-1 cells do indeed produce factor(s) that promote repopulation. In addition, we have demonstrated that tumour cell repopulation is inhibited by the PAFR antagonist WEB 2170 as well as several phomactin natural products (albeit at modest potencies), with phomactin R showing superior results. Given the emerging significance of PAFR antagonists in inhibiting irradiated tumour cell repopulation in in vivo systems ${ }^{39}$, our observations here warrant additional study. Our future work will seek to make more significant disruptive changes to the structures of the phomactins to gain more insight into their SARs.

\section{Conclusions}

Natural product chemistry remains critical to the discovery of small molecules that possess unique bioactivities. By pursuing the isolation, chemical synthesis and biological evaluation of natural products and their derivatives in tandem, a more effective and comprehensive approach to identifying small molecules that possess significant bioactivity can be established. We have demonstrated the success of this approach with the phomactin diterpenoids. Novel congeners of the phomactins $(\mathrm{Q}-\mathrm{V}, \mathbf{1}-\mathbf{6})$ were obtained from media extracts of Biatriospora sp. (CBMAI 1333), a fungus isolated from a marine sponge collected off the Brazilian coastline. A bioinspired chemical synthesis approach enabled the efficient preparation of some of these newly isolated phomactin natural products as well as previously isolated congeners that are representative of each phomactin subclass. Our chemical syntheses feature the use of $(S)$-(+)-carvone as a starting material, a unique Rh-catalysed cyclobutanol opening/protonation that formally achieves a rearrangement of the carvone framework to incorporate the isopropenyl substituent, and late-stage diversification to access a range of phomactin congeners. Biological activity investigations revealed notable PAFR antagonist activity for several of the phomactin natural products. Although no clear SAR correlations emerged, in the majority of cases, the PAFR antagonist activity correlates with the ability of these molecules to inhibit the repopulation of tumour cells that have undergone gamma irradiation.

Several aspects of this work would not have been possible had the isolation, synthesis and bioactivity assays not been conducted in tandem. For example, we subjectively chose to pursue a bioinspired synthetic strategy with an eye towards gaining access to a wide range of phomactin congeners, including the novel isolates reported here. In doing so, chemical synthesis facilitated their unambiguous structural characterization. In addition, our PAFR antagonist activity evaluation of the phomactins at an early stage inspired the novel tumour cell repopulation studies of the phomactins. Finally, unambiguous structural elucidation of phomactin $\mathrm{T}$ was achieved in a case where the material isolated from the natural source had decomposed and the material required to complete structural analysis was only available through synthesis. Many such scenarios where only a small amount of a secondary metabolite is isolated, or decomposes, exist. In these cases, natural products that may possess useful bioactivity go unreported or may be mischaracterized. Here, our collaboration yielded additional material through synthesis to complete unambiguous structural characterization.

Finally, several additional studies have been inspired by our collaboration. For example, it can be envisioned that some of the latestage synthetic intermediates en route to our completed bioinspired chemical syntheses of the phomactins may themselves be secondary metabolites that exist in the producing organism but are yet to be isolated. We are therefore re-examining the cultures of Biatriospora sp. (CBMAI 1333) at different time points to see if some of these synthetic intermediates can be detected as secondary metabolites. With authentic samples now available from chemical synthesis, detection of the natural isolates may be accelerated, thus providing insight into the range of naturally occurring phomactin natural products. Finally, the preliminary biological results described here portend excellent bioactivity for derivatives of the phomactins that may be accessed through chemical synthesis. These studies (both in vitro and in vivo) constitute a major portion of our future studies of the phomactins.

\section{Methods}

Assay of PAFR antagonistic activity. Immortalized human epithelial cells (KBMs) that do not express PAFR were transfected with the PAF-receptor (KBP). Then, $2 \times 10^{5} \mathrm{KBP}$ cells were plated in 24-well plates in Dulbecco's modified Eagle's medium (DMEM, GIBCO) supplemented with 10\% FCS, penicillin $\left(100 \mathrm{U} \mathrm{ml}^{-1}\right)$ and streptomycin $\left(100 \mu \mathrm{g} \mathrm{ml}^{-1}\right)$. After $24 \mathrm{~h}$, these cells were stimulated with $10 \mathrm{nM}$ methylcarbamyl PAF C16 (cPAF). After $6 \mathrm{~h}$, supernatants were collected for IL-8 measurement (readout of PAFR activation ${ }^{40}$ ) using BD OptEIA ELISA sets (BD Biosciences). Control PAFR antagonist WEB 2170 and phomactin samples were added to the KBP cells, in doses ranging from 1 to $100 \mu \mathrm{M}$ (in DMSO), $30 \mathrm{~min}$ before $\mathrm{CPAF}$ addition. We established $10 \mathrm{nM}$ as the concentration of cPAF that induces $50 \%$ of the maximal response. This concentration of $\mathrm{CPAF}$ was then used to determine the concentration of WEB 2170 required to cause $50 \%$ inhibition $\left(\mathrm{IC}_{50}\right.$ ) (S40). The same concentration of CPAF was then used to calculate the $\mathrm{IC}_{50}$ of the phomactins (Table 1). Of note, cPAF was used in this study because it is resistant to PAF acetyl hydrolase, a cellular enzyme that degrades PAF.

Tumour cell repopulation assay. The method used has been described previously ${ }^{39}$. The TC-1 cell line (lung epithelium of murine carcinoma) was used. For the assay, a layer of irradiated ( $8 \mathrm{~Gy}$ ) or non-irradiated TC-1 cells that express PAFR (TC-1 cells, $2 \times 10^{5}$ ) were co-cultured with $10^{3}$ luciferase-labelled TC-1 cells (TC-1 Fluc ${ }^{+}$). Cell irradiation was conducted using an IBL 136 cell and animal gamma radiator at a dose rate of $251.7 \mathrm{cGy} \mathrm{min}^{-1}$. The $8 \mathrm{~Gy}$ dose was selected on the basis of previous studies and represents the best dose for induction of repopulation phenomena in vitro ${ }^{39,41}$. After nine days, the proliferation of the TC-1 Fluc ${ }^{+}$cells was evaluated as relative luminescence units (RLU) (S41 A). The TC-1 Fluc ${ }^{+}$cells grew significantly faster when seeded onto irradiated TC-1 than when seeded onto non-irradiated cells (S41 B). Notably, when seeded onto nonirradiated cells, the growth of the TC-1 Fluc ${ }^{+}$cells was very slow (fluorescence levels were below detection). Fluc ${ }^{+} \mathrm{TC}-1$ cells were treated with PAFR antagonists or phomactins, $30 \mathrm{~min}$ before irradiation and each 2 days until 9 days of culture. Results are presented as RLU or \% inhibition of RLU relative to the non-treated group. Of note, all cell lines used in this work were regularly tested for Mycoplasma and were free of this contaminant.

Cell viability assay. The concentrations of phomactins used in this study were all assayed for viability to avoid using concentrations that are toxic to the cells. We measured LDH released into the media from damaged cells as a biomarker for cellular cytotoxicity and cytolysis. A colorimetric viability assay using commercially available kits (BioClin) was used according to the manufacturer's instructions. 
Statistical analysis. Data were analysed using GraphPad Prism 4.0 software. For each individual cell-based assay, compounds were run in duplicate (binding) or triplicate and normalized to the untreated cells. Dose-response curves were generated using three-parameter nonlinear regression analysis. Data are from $n \geq 3$ independent experiments and are presented as mean \pm s.e.m. or as indicated in the legend.

Materials. Methylcarbamyl PAF (cPAF) was obtained from Cayman Chemical. The reference compound WEB 2170 was obtained from Boehringer Ingelheim, Pharma $\mathrm{KG}$ and was prepared in DMSO as a $5 \mathrm{mM}$ stock solution.

Reporting Summary. Further information on experimental design is available in the Nature Research Reporting Summary linked to this article.

Data availability. The characterization data for all new chemical compounds are provided in the Supplementary Information. The.cif file for phomactin S (3) has been deposited at the Cambridge Crystallographic Data Centre (CCDC 1830519).

Received: 25 October 2017; Accepted: 15 May 2018;

Published online: 30 July 2018

\section{References}

1. Maier, M. E. Structural revisions of natural products by total synthesis. Nat. Prod. Rep. 26, 1105-1124 (2009).

2. Wilson, R. M. \& Danishefsky, S. J. Small molecule natural products in the discovery of therapeutic agents: the synthesis connection. J. Org. Chem. 71, 8329-8351 (2006).

3. Maier, M. E. Design and synthesis of analogues of natural products. Org. Biomol. Chem. 13, 5302-5343 (2015).

4. Newman, D. J. \& Cragg, G. M. Natural products as sources of new drugs from 1981 to 2014. J. Nat. Prod. 79, 629-661 (2016).

5. Sugano, M. et al. Phomactin A: a novel PAF antagonist from a marine fungus Phoma sp. J. Am. Chem. Soc. 113, 5463-5464 (1991).

6. Sugano, M. et al. Phomactins, novel PAF antagonists from marine fungus Phoma sp. J. Org. Chem. 59, 564-569 (1994).

7. Sugano, M. et al. Phomactin E, F, and G: new phomactin-group PAF antagonists from a marine fungus Phoma sp. J. Antibiot. 48, 1188-1190 (1995).

8. Chu, M. et al. A novel class of platelet activating factor antagonists from Phoma sp. J. Antibiot. 46, 554-563 (1993).

9. Koyama, K. et al. Phomactin $\mathrm{H}$, a novel diterpene from an unidentified marine-derived fungus. Tetrahedron Lett. 45, 6947-6948 (2004).

10. Ishino, M. et al. Phomactin I, 13-epi-phomactin I, and phomactin J, three novel diterpenes from a marine-derived fungus. Tetrahedron $\mathbf{6 6}$ 2594-2597 (2010).

11. Ishino, M. et al. Phomactins K-M, three novel phomactin-type diterpenes from a marine-derived fungus. Tetrahedron 68, 8572-8576 (2012).

12. Ishino, M. et al. Three novel phomactin-type diterpenes from a marinederived fungus. Tetrahedron Lett. 57, 4341-4344 (2016)

13. Passarini, M. R. Z., Santos, C., Lima, N., Berlinck, R. G. S. \& Sette, L. D. Filamentous fungi from the Atlantic marine sponge Dragmacidon reticulatum. Arch. Microbiol. 195, 99-111 (2013).

14. Goldring, W. P. D. \& Pattenden, G. The phomactins. A novel group of terpenoid platelet activating factor antagonists related biogenetically to the taxanes. Acc. Chem. Res. 39, 354-361 (2006).

15. Prescott, S. M., Zimmerman, G. A., Stafforini, D. M. \& McIntyre, T. M. Platelet-activating factor and related lipid mediators. Annu. Rev. Biochem. 69, 419-445 (2000).

16. Onuchic, A. C. et al. Expression of PAFR as part of a pro-survival response to chemotherapy: a novel target for combination therapy in melanoma. Mediat. Inflamm. 2012, 175408 (2012).

17. Sahu, R. P. et al. Radiation therapy generates platelet-activating factor agonists. Oncotarget 7, 20788-20800 (2016).

18. Ciesielski, J. \& Frontier, A. The phomactin natural products from isolation to total synthesis: a review. Org. Prep. Proced. Int 46, 214-251 (2014).

19. Goldring, W. P. D.., \& Pattenden, G.. A total synthesis of phomactin. Chem. Commun. 2002, 1736-1737 (2002)

20. Goldring, W. P. D. \& Pattenden, G. Total synthesis of ( \pm )-phomactin G, a platelet activating factor antagonist from the marine fungus Phoma sp Org. Biomol. Chem. 2, 466-473 (2004).

21. Huang, J., Wu, C. \& Wulff, W. D. Total synthesis of ( \pm )-phomactin B2 via an intramolecular cyclohexadienone annulation of a chromium carbene complex. J. Am. Chem. Soc. 129, 13366-13367 (2007).

22. Tang, Y., Cole, K. P., Buchanan, G. S., Li, G. \& Hsung, R. P. Total synthesis of phomactin A. Org. Lett. 11, 1591-1594 (2009).

23. Miyaoka, H., Saka, Y., Miura, S. \& Yamada, Y. Total synthesis of phomactin D. Tetrahedron Lett. 37, 7107-7110 (1996).

24. Mohr, P. J. \& Halcomb, R. L. Total synthesis of (+)-phomactin A using a B-alkyl Suzuki macrocyclization. J. Am. Chem. Soc. 125, 1712-1713 (2003).
25. Tokiwano, T., Fukushi, E., Endo, T. \& Oikawa, H. Biosynthesis of phomactins: common intermediate phomactatriene and taxadiene. Chem. Commun. 1324-1325 (2004).

26. Tokiwano, T. et al. Proposed mechanism for diterpene synthases in the formation of phomactatriene and taxadiene. Org. Biomol. Chem. 3, 2713-2722 (2005).

27. Masarwa, A., Weber, M. \& Sarpong, R. Selective C-C and C-H bond activation/cleavage of pinene derivatives: synthesis of enantiopure cyclohexenone scaffolds and mechanistic insights. J. Am. Chem. Soc. 137, 6327-6334 (2015)

28. Bermejo, F. A. et al. Ti(III)-promoted cyclizations. Application to the synthesis of (E)-endo-bergamoten-12-oic acids. Moth oviposition stimulants isolated from Lycopersicon hirsutum. Tetrahedron 62, 8933-8942 (2002).

29. Murakami, M., Makino, M., Ashida, S. \& Matsuda, T. Construction of carbon frameworks through $\beta$-carbon elimination mediated by transition metals. Bull. Chem. Soc. Jpn 79, 1315-1321 (2006).

30. Cramer, N. \& Seiser, T. $\beta$-Carbon elimination from cyclobutanols: a clean access to alkylrhodium intermediates bearing a quaternary stereogenic center. Synlett 449-460 (2011).

31. Lipshutz, B. H. \& Miller, T. A. Deprotection of 'SEM' ethers: a convenient, general procedure. Tetrahedron Lett. 30, 7149-7152 (1989).

32. Nicolaou, K. C. \& Harrison, S. T. Total synthesis of abyssomicin C and atrop-abyssomicin C. Angew. Chem. Int. Ed. 45, 3256-3260 (2006).

33. Catino, A. J., Forslund, R. E. \& Doyle, M. P. Dirhodium(II) caprolactamate: an exceptional catalyst for allylic oxidation. J. Am. Chem. Soc. 126, 13622-13623 (2004)

34. Honda, T. \& Mizutani, H. Regioselective ring-opening of 2,3-epoxy alcohols with tetramethylammonium triacetoxyborohydride. Heterocycles $\mathbf{4 8}$, 1753-1757 (1998).

35. Evans, D. A., Chapman, K. T. \& Carreira, E. M. Directed reduction of $\beta$-hydroxy ketones employing tetramethylammonium tracetoxyborohydride. J. Am. Chem. Soc. 110, 3560-3578 (1988).

36. Jancar, S. \& Chammas, R. PAF receptor and tumor growth. Curr. Drug Targets 15, 982-987 (2014).

37. Bussolati, B. et al. PAF produced by human breast cancer cells promotes migration and proliferation of tumor cells and neo-angiogenesis. Am. J. Pathol. 157, 1713-1725 (2000).

38. Chan, F. K.-M., Moriwaki, K. \& De Rosa, M. J. in Immune Homeostasis. Methods and Protocols Vol. 979 (eds Snow, A. \& Lenardo, M.) 65-70 (Humana, Totowa, 2013).

39. da Silva-Jr, I. A., Chammas, R., Lepique, A. P. \& Jancar, S. Platelet-activating factor (PAF) receptor as a promising target for cancer cell repopulation after radiotherapy. Oncogenesis 6, e296 (2017).

40. Rios, F. J. O., Koga, M. M., Ferracini, M. \& Jancar, S. Co-stimulation of PAFR and CD36 is required for oxLDL-induced human macrophages activation. PLoS ONE 7, e36632 (2012).

41. Huang, Q. et al. Caspase 3-mediated stimulation of tumor cell repopulation during cancer radiotherapy. Nat. Med. 17, 860-866 (2011).

\section{Acknowledgements}

The authors thank K. Koyama (Meiji Pharmaceutical University) for providing spectral data for phomactin P and S. Dreher and A. Buevich (Merck Pharmaceuticals) for the ${ }^{1} \mathrm{H}$ NMR spectrum of Sch 49027. R.S. thanks the National Science Foundation (CHE1566430) for financial support. Y.K. thanks the Japan Society for the Promotion of Science (JSPS) for an Overseas Research Fellowship. P.R.L. thanks the National Science Foundation for a graduate research fellowship. S.C. acknowledges a National Science and Engineering Council-Canada (NSERC) Postdoctoral Fellowship. R.G.S.B and J.R.G. thank FAPESP (Fundaçao de Amparo a Pesquisa de São Paulo) for financial support (BIOTA-BIOprospecTA 2013/50228-8, 2015/01017-0 and 2017/06014-4) and K.J.N. thanks CNPq for a PhD scholarship. S.J. and I.A.S.J. are grateful to FAPESP for financial support and a graduate research fellowship. N.N. thanks JSPS for a travel fellowship. K.B. is grateful to the Amgen Scholar Program (UC Berkeley) for support. R.J.A. thanks the NSERC for funding. The KBP cells were supplied by J.B. Travers (Indiana University, Indianapolis, IN) and the TC-1 cell line was donated to us by T.-C. Wu (Johns Hopkins, Baltimore). The authors thank K. Owens for insightful discussions regarding the structure of Sch 49027.

\section{Author contributions}

R.G.S.B. (isolation and identification), Y.K. (chemical synthesis), R.S. (chemical synthesis) and S.J. (biological assays) wrote each of the corresponding sections and R.S. composed the manuscript. P.R.L., S.C. and R.S. conceived the general plan for the chemical synthesis of phomactin R. Y.K. and R.S. designed the plan for the chemical syntheses of phomactins A, K, P, T and Sch 49027. P.R.L. and S.C. carried out the initial studies on the cyclobutanol opening, cyclohexenone functionalization, and macrocyclization that provided a part of the basis of the reported syntheses. Y.K. conducted the chemical reactions reported herein and compiled the Supplementary Information. N.N. conducted the large-scale preparation of $\mathbf{1 8}$ and $\mathbf{2 0}$ to support frontline synthetic studies. A.R. and K.B. carried out exploratory studies on the cyclobutanol 
intermediates and subsequent functionalizations. K.J.N. isolated the new phomactins and elucidated their structures jointly with J.R.G. V.M.D. performed X-ray diffraction analysis. A.G.F., D.E.W. and R.J.A. provided support and performed NMR analyses of the new phomactins. L.D.S. provided and identified the fungal strain. I.A.S.J. and S.J. performed the assays for PAFR antagonistic activity and the repopulation assays with cancer cell lineages.

\section{Competing interests}

The authors declare no competing interests.

\section{Additional information}

Supplementary information is available for this paper at https://doi.org/10.1038/ s41557-018-0084-X.

Reprints and permissions information is available at www.nature.com/reprints. Correspondence and requests for materials should be addressed to S.J. or R.G.S.B. or R.S.

Publisher's note: Springer Nature remains neutral with regard to jurisdictional claims in published maps and institutional affiliations. 


\section{Reporting Summary}

Nature Research wishes to improve the reproducibility of the work that we publish. This form provides structure for consistency and transparency in reporting. For further information on Nature Research policies, see Authors \& Referees and the Editorial Policy Checklist.

\section{Statistical parameters}

When statistical analyses are reported, confirm that the following items are present in the relevant location (e.g. figure legend, table legend, main text, or Methods section).

n/a Confirmed

$\bigotimes$ The exact sample size $(n)$ for each experimental group/condition, given as a discrete number and unit of measurement

$\searrow$ An indication of whether measurements were taken from distinct samples or whether the same sample was measured repeatedly

$\triangle$ The statistical test(s) used AND whether they are one- or two-sided

$\triangle$ Only common tests should be described solely by name; describe more complex techniques in the Methods section.

Х $\square$ A description of all covariates tested

$\square$ \ A description of any assumptions or corrections, such as tests of normality and adjustment for multiple comparisons A full description of the statistics including central tendency (e.g. means) or other basic estimates (e.g. regression coefficient) AND variation (e.g. standard deviation) or associated estimates of uncertainty (e.g. confidence intervals)

For null hypothesis testing, the test statistic (e.g. $F, t, r$ ) with confidence intervals, effect sizes, degrees of freedom and $P$ value noted Give $P$ values as exact values whenever suitable.

Х $\square$ For Bayesian analysis, information on the choice of priors and Markov chain Monte Carlo settings

Х $\square$ For hierarchical and complex designs, identification of the appropriate level for tests and full reporting of outcomes

Х $\square$ Estimates of effect sizes (e.g. Cohen's $d$, Pearson's $r$ ), indicating how they were calculated

$\varnothing$ Clearly defined error bars

$\triangle$ State explicitly what error bars represent (e.g. SD, SE, CI)

Our web collection on statistics for biologists may be useful.

\section{Software and code}

\section{Policy information about availability of computer code}

Data collection IL-8 BD OptEIA ${ }^{\mathrm{TM}}$ ELISA, LDH (lactate Dehydrogenase assay) and RLU (Relative Luminescence Units) was measured on a microtiter plate reader (SpectraMax 190; Molecular Devices) using SoftMax Pro Software version 2.0.

Data analysis

Data from biological assays were analyzed using GraphPad Prism software (San Diego, California) version 4.0.

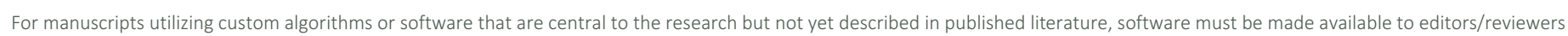

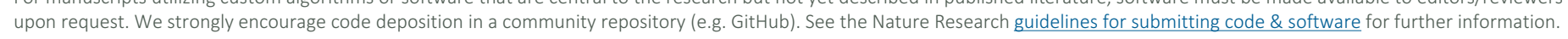

\section{Data}

Policy information about availability of data

All manuscripts must include a data availability statement. This statement should provide the following information, where applicable:

- Accession codes, unique identifiers, or web links for publicly available datasets

- A list of figures that have associated raw data

- A description of any restrictions on data availability

The authors declare that all data supporting the findings of this study are available in the main manuscript and in the supplementary information files. 


\section{Field-specific reporting}

Please select the best fit for your research. If you are not sure, read the appropriate sections before making your selection.

\section{$\bigotimes$ Life sciences $\quad \square$ Behavioural \& social sciences}

For a reference copy of the document with all sections, see nature.com/authors/policies/ReportingSummary-flat.pdf

\section{Life sciences}

\section{Study design}

All studies must disclose on these points even when the disclosure is negative.

Sample size

Sample size was based on experience in prior studies and sized to allow significance in biologically relevant effect sizes. We did not use a statistical method to predetermine sample size. The in vitro data were repeated independently at least three times to ensure reproducible conclusions. In each experiment, three biological replicates per condition or treatment group were collected for analyses. The exact number of repetitions is stated in the legend of each figure.

Data exclusions

No data were exluded from the analyses.

Replication

Differences between control and treatment showed consistent and reproducible results across our study. For in vitro experiments, multiple biological replicates were used for each treatment group. The exact number of independent experiments conducted for each experiment are described in the legends of figures.

Randomization The samples were allocated in experimental or control groups randomly.

Blinding

Blinding was not relevant in the experiments perfomed in this manuscript.

\section{Materials \& experimental systems}

Policy information about availability of materials

$\mathrm{n} / \mathrm{a}$ Involved in the study

$\square \bowtie$ Unique materials

$\square$ \ Antibodies

$\square$ Eukaryotic cell lines

$\searrow \square$ Research animals

Х Human research participants

Unique materials

Obtaining unique materials

The fungus strain Biatriospora sp. CBMAI 1333 is available upon request to the authors R. G. S. B. or L. D. S.

Antibodies

Antibodies used

Anti human IL-8 OptEIA ${ }^{\text {TM }}$ ELISA (BD Bioscience, catalog number 555244, lot number 5044720). Capture and detection antibody were used at 1:250 recommended dilution as describe in Technical Data Sheet on manufacturer's website http:// www.bdbiosciences.com/ds/pm/tds/555244.pdf.

Validation

All the antibodies used in this stud were validated and tested by BD Bioscience. Data are available on manufacturer's website.

Eukaryotic cell lines

Policy information about cell lines

Cell line source(s)

TC-1 cells, an established murine SCC line, were kindly donated by Dr. T.-C. Wu (John Hopkins, Baltimore) and KBM/KBP cells from Dr. J. B. Travers (Department of Dermatology, Indiana University School of Medicine, Indianapolis, IN, USA).

Authentication

None of the cell lines used were authenticaded.

Mycoplasma contamination

All cell lines were tested for Mycoplasma contamination and were negative.

Commonly misidentified lines (See ICLAC register)

No commonly misidentified cell lines were used in this study. 
Method-specific reporting

$\mathrm{n} / \mathrm{a} \mid$ Involved in the study

Х $\square$ ChIP-seq

Х $\square$ Flow cytometry

\ $\square$ Magnetic resonance imaging 